\title{
Giving subjects the eye and showing them the finger: Socio-biological cues and saccade generation in the anti-saccade task
}

\author{
Nicola J Gregory, Timothy L Hodgson§ \\ Washington Singer Laboratories, Psychology, College of Life and Environmental Sciences, University \\ of Exeter, Exeter EX4 4QG, UK; §School of Psychology, University of Lincoln, Brayford Pool, Lincoln \\ LN6 7TS, UK; e-mail: tlhodgson@lincoln.ac.uk \\ Received 27 July 2011, in revised form 16 January 2012
}

\begin{abstract}
Pointing with the eyes or the finger occurs frequently in social interaction to indicate direction of attention and one's intentions. Research with a voluntary saccade task (where saccade direction is instructed by the colour of a fixation point) suggested that gaze cues automatically activate the oculomotor system, but non-biological cues, like arrows, do not. However, other work has failed to support the claim that gaze cues are special. In the current research we introduced biological and non-biological cues into the anti-saccade task, using a range of stimulus onset asynchronies (SOAs). The anti-saccade task recruits both top-down and bottom - up attentional mechanisms, as occurs in naturalistic saccadic behaviour. In experiment 1 gaze, but not arrows, facilitated saccadic reaction times (SRTs) in the opposite direction to the cues over all SOAs, whereas in experiment 2 directional word cues had no effect on saccades. In experiment 3 finger pointing cues caused reduced SRTs in the opposite direction to the cues at short SOAs. These findings suggest that biological cues automatically recruit the oculomotor system whereas nonbiological cues do not. Furthermore, the anti-saccade task set appears to facilitate saccadic responses in the opposite direction to the cues.
\end{abstract}

\section{Introduction}

We interact with the people around us by both verbal and non-verbal means. Although we may regard language as the primary means of communication, we also express our emotions and intentions by gestures and through the movements of our eyes. For example, if you are at a party and are conversing with a stranger who repeatedly glances towards the door, you may be warranted in thinking that he/she is planning to escape your company at the first available opportunity or that he/she is expecting someone more interesting to imminently arrive. Similarly, if you were to stop on any street and ask for directions, it would be unlikely that the detailed reply you receive will not be accompanied by pointing gestures in the direction in which you are being advised to travel.

Previous research has suggested that the direction of eye gaze of others may constitute a special example of a directional signal, due to its importance in indicating intention and attention in the social world. Evidence from covert spatial cueing paradigms has suggested that the perception of non-informative averted eye gaze cues results in an automatic shift of attention in the cued direction, with targets at the cued location detected more quickly than those appearing at an uncued location (eg Bonato et al 2009; Driver et al 1999; Friesen and Kingstone 1998; Quadflieg et al 2004; Ristic et al 2002). Furthermore, it has been shown that gaze orienting persists even when the cues point in the wrong direction for the target on the majority of trials (Driver et al 1999; Friesen et al 2004). It would appear that gaze cues are not alone in automatically influencing visual attention, however. Recent work has suggested that task-irrelevant finger-pointing cues also have apparently obligatory effects on covert attention, causing facilitation of target detection in the direction indicated by the cues (Ariga and Watanabe 2009; Sato et al 2010; Tomonaga and Imura 2009). However, a raft of evidence has been 
presented which demonstrates that these effects on covert attention are not restricted to biological cues. Rather, non-biological arrow cues have been repeatedly shown to shift covert attention when they are task-irrelevant and to-be-ignored (eg Bonato et al 2009; Gibson and Bryant 2005; Hommel et al 2001; Ristic and Kingstone 2006; Ristic et al 2002; Tipples 2002, 2008). In addition, directional word cues which are non-predictive of target location and are task-irrelevant have also been reported to result in covert attentional shifts as shown by enhanced detection of peripheral stimuli appearing at cued locations relative to uncued locations (Ho and Spence 2006; Hommel et al 2001).

The literature on eye movement responses to biological and non-biological cues is relatively sparse in comparison to that investigating covert attention, and has focused almost exclusively on comparing eye gaze and arrow cues. A study by Ricciardelli et al (2002) suggested that averted eye gaze, but not non-biological arrows, caused observers to automatically execute eye movements in the direction of the cues, despite the observers being explicitly instructed to ignore them. This finding formed the basis of what has subsequently been referred to as the gaze imitation hypothesis. However, no studies examining eye movements in response to gaze and arrow cues have managed to replicate the results of Ricciardelli et al. Indeed, Kuhn and colleagues (Kuhn and Benson 2007; Kuhn and Kingstone 2009; Kuhn et al 2010) have shown that gaze and arrow cues have equivalent effects on both saccadic reaction times and error rates within the same voluntary saccade task used by Ricciardelli et al, where participants have to make a saccade in the direction indicated by the colour change of a fixation cross while task-irrelevant cues are presented on screen.

To our knowledge, to date no studies have investigated the influence of fingerpointing cues on oculomotor responses. However, some work has been presented which demonstrates that directional word cues can automatically influence the saccadic system. Hodgson et al (2009) employed an oculomotor Stroop task where participants were required to make a saccade to one of four coloured patches on a computer screen. The correct direction of the saccade was determined by the 'ink' colour of a centrally presented colour or direction word, whilst the to-be-ignored identity of the word could be congruent or incongruent with the ink colour. These authors found that when the ink and identity of the word were incongruent, participants were slower to correctly initiate a saccade to the correct colour patch, but also that they made error saccades towards the patch indicated by the word identity. These findings suggest that the semantic meaning of words can be processed automatically, even when they are to be ignored, generating a corresponding oculomotor programme matching the meaning of the word.

The results of Kuhn and colleagues (Kuhn and Benson 2007; Kuhn and Kingstone 2009; Kuhn et al 2010) and those of the multitude of studies which have found no differences between the effects of biological and non-biological cues on covert attention, are somewhat counterintuitive. Eye gaze is clearly an important aid in social interaction which is processed and interpreted without effort in everyday life, with some evidence suggesting that gaze following may even be present from birth (Farroni et al 2002, 2004). Arrows and written directional words on the other hand are clearly non-biological, being used to direct our action and attention in the absence of human input. Moreover, there can be no innate ability for detecting and orienting to arrows or words as these are human constructs. Therefore, differences between the effects of biological and non-biological cues on saccadic responses could be reasonably expected, but why is empirical evidence for such differences proving so elusive?

A possible explanation for this lack of differentiation between the saccadic orienting effects, elicited by gaze and arrow cues in particular, may lie in the choice of paradigm used to study the cues. In the voluntary saccade task participants are required to pay particular attention to the fixation cross in order to detect its colour change, whilst at 
the same time being instructed to ignore the cues appearing at the same location. This may present a possible confound, as the cues may be more likely to be automatically processed, due to increased focused attention at the fixation location than a static fixation point. Further, the voluntary saccade task is rather simplistic investigating, as the name suggests, only volitional saccade generation. A more appropriate paradigm with which to study the influence of cues on saccadic behaviour may be the anti-saccade task. First described by Hallett (Hallett 1978; Hallett and Adams 1980), the anti-saccade task requires participants to make a saccade in the opposite direction to the onset of a peripheral stimulus. In order to successfully execute an anti-saccade, the execution of a reflexive saccade towards the 'target' must first be suppressed and second a saccade in the opposite direction has to be generated (Everling and Fischer 1998; Guitton et al 1985; Munoz and Everling 2004). Errors, where participants incorrectly look towards the peripheral stimulus, are common in neurologically healthy controls, occurring on about $20 \%$ of trials (Hutton 2008). The suppression of such reflexive saccades requires substantially more cognitive effort than is required to complete the voluntary saccade task or the pro-saccade task (where a saccade is made towards, rather than away from, a target). The interaction between bottom-up and top-down control of attention in the anti-saccade task more closely resembles the competing influences on saccadic orienting which occur in naturalistic settings and in this sense the task provides a more ecologically valid paradigm with which to study the influence of task-irrelevant cues on eye movements than either the voluntary saccade task or the pro-saccade task.

A study by Koval et al (2005) utilised the anti-saccade task to examine the effects of task-irrelevant gaze cues on eye movements. Rather than demonstrating that participants were faster to correctly respond in the direction of the cues, a finding which would have supported the gaze imitation hypothesis, Koval et al found that saccadic reaction time (SRT) was reduced on congruent trials relative to incongruent, and that error rates were higher when cue and target were incongruent. In other words, participants' saccadic responses, reflexive (reflected in error rates) and voluntary (as indicated by correct SRT) were facilitated in the opposite direction to the cues. These authors did not provide a comparison non-biological cue however, leaving open the possibility that similar results may be found with cues such as arrows. What the results of this study do suggest is that, if the experimental task can unexpectedly influence the direction of facilitation of responses to gaze cues, it may also uncover differences between biological gaze and finger pointing cues and non-biological cues such as arrows and words.

The current research examined the influence of task-irrelevant gaze, arrow, word, and finger pointing cues on saccadic responses in the anti-saccade task. Experiment 1 set out to extend the findings of Koval et al (2005) that, in this task, participants' responses are enhanced in the opposite direction to gaze cues, by comparing gaze and arrow cues within the same anti-saccade task. It was anticipated that the use of the more cognitively complex paradigm might highlight differences between the biological and non-biological cues which have hitherto remained elusive. In experiments 2 and 3, directional word cues (LEFT and RIGHT) and photographic finger pointing cues were presented, respectively, within the same anti-saccade task used in experiment 1 . As with experiment 1, the increased demands of the anti-saccade task might be expected to over-ride the automatic influence of the word cues seen in Hodgson et al's (2009) version of the Stroop task. Furthermore, if the biological nature of cues is important in producing saccadic orienting, then finger pointing cues may influence responses similarly to gaze cues, with arrows and words affecting saccadic orienting to a lesser degree. 


\section{Experiment 1}

\subsection{Material and methods}

2.1.1 Participants. Fourteen psychology undergraduates (thirteen female) aged between 18 and 43 years $(M=23.00$ years, $\mathrm{SD}=7.93$ years $)$ took part in this experiment in exchange for course credit. All participants had either normal or corrected-to-normal vision. Written informed consent was given by all participants, and ethical approval for this experiment was granted by the Ethics Committee of the School of Psychology, University of Exeter. The research was carried out in accordance with The Code of Ethics of the World Medical Association (Declaration of Helsinki).

2.1.2 Apparatus and stimuli. Stimuli were presented on a 19 inch Iiyama MA901U Vision Master Pro 452 CRT monitor connected to a Dell Optiplex GX260 desktop computer. Participants sat approximately $60 \mathrm{~cm}$ from the monitor. Eye-movement recording was carried out by means of the head-mounted EyeLink II system (SR Research, Canada), connected to a second Dell Optiplex GX260 desktop computer with an Ethernet link connecting the host computer to the display computer. The EyeLink II tracked pupil movement at a rate of $500 \mathrm{~Hz}$. The position of the head was monitored by an integral camera, mounted centrally on the headband, which recorded illumination from four infrared emitting LED markers, mounted on the edges of the display computer's screen, which compensated for small head movements. Images displayed were prepared with Corel Paint Shop Pro X image editing software. The arrow cues stimuli were UK road signs for 'keep left' and 'keep right', comprised of a blue circle with a white arrow. The neutral condition was represented by the UK road sign for 'mini roundabout', consisting of three small white arrows forming a circle, again on a blue background. The arrow cues measured $4.45 \mathrm{deg}$ of visual angle. The gaze cues were cropped colour photographs of a Caucasian male face, showing just the eye region. The left version of the cue was identical to the right version but with the sclera and pupil area only displayed in mirror image. The neutral condition displayed the same model with eyes closed. The cues measured $5.52 \mathrm{deg}$ of visual angle. The cue stimuli used in experiments $1-3$ can be seen in figure 1 .

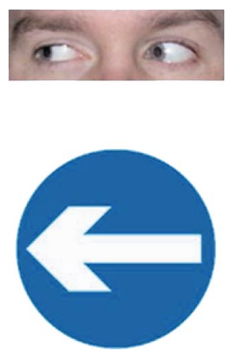

(a)

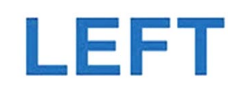

RIGHT
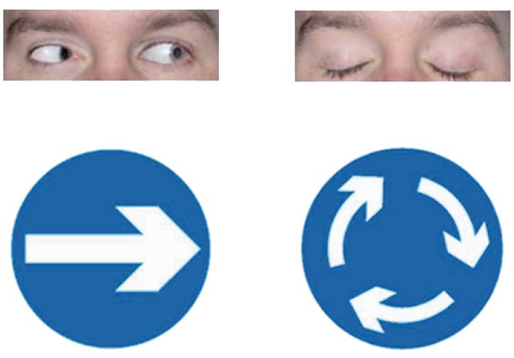

$\mathbf{X X X X}$

(b)

(c)
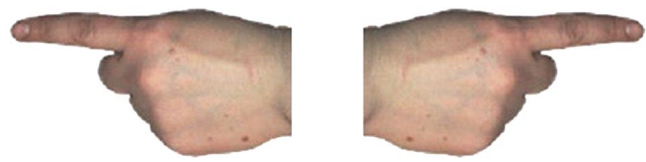

Figure 1. [In colour online, see http://dx.doi.org/10.1068/p7085] (a) Gaze and arrow cue stimuli used in experiment 1; (b) word cues used in experiment 2; and (c) finger pointing cues used in experiment 3. Images are not drawn to scale. 
2.1.3 Procedure. The experiment was blocked by the factor cue type (arrow or eye gaze). The order in which the cue types were presented was counterbalanced between subjects to prevent any order effects from emerging. Each cue type condition contained 180 trials, split into three blocks, so that each participant completed 360 trials in total. At the beginning of each set of three blocks a calibration procedure was performed in order to map raw data into eye gaze position. The participant fixated a dot displayed on the display computer as it moved in steps around the periphery of the screen. In addition to this calibration, at the beginning of each block of 60 trials, and every 10 trials thereafter, a drift-correct procedure was carried out in order to account for any headband slippage that may have occurred. The participant fixated a single central dot and the difference between that position and that expected from the calibration procedure was computed.

All trials were presented on a white background. Each trial began with the presentation of a black central fixation cross subtending $0.95 \mathrm{deg}$ of visual angle, for the duration of $1000 \mathrm{~ms}$. Following this, the cue, which could be pointing left or right and could be congruent, incongruent, or neutral in respect to the 'target', was presented. (Note that congruency refers to the cue- 'target' congruency.) After a stimulus onset asynchrony (SOA) of 100, 300, or $800 \mathrm{~ms}$, the fixation cross was removed, simultaneously with the onset of the target, a black dot with a diameter of $1.26 \mathrm{deg}$ of visual angle. The dot could appear vertically centred at either the left or right of the screen at an eccentricity of $14.75 \mathrm{deg}$ of visual angle, where it remained for $2000 \mathrm{~ms}$. The target dot appeared on the left and right sides of the screen with equal probability and for a total of 90 left and 90 right presentations for each set of three blocks. The participants were instructed to maintain fixation on the central cross and ignore the road signs or eyes until the dot appeared, whereupon they were to make a saccade in the opposite direction to the dot as quickly as possible. Participants were informed that the direction to which the arrows or eyes pointed in no way predicted the likely position of the target dot. The direction, congruency, and SOA of the cues were presented randomly. An interval of $1000 \mathrm{~ms}$ separated each trial. Figure 2 shows the procedure for a congruent gaze trial and an incongruent arrow trial. The correct response is in the opposite direction to the 'target'.

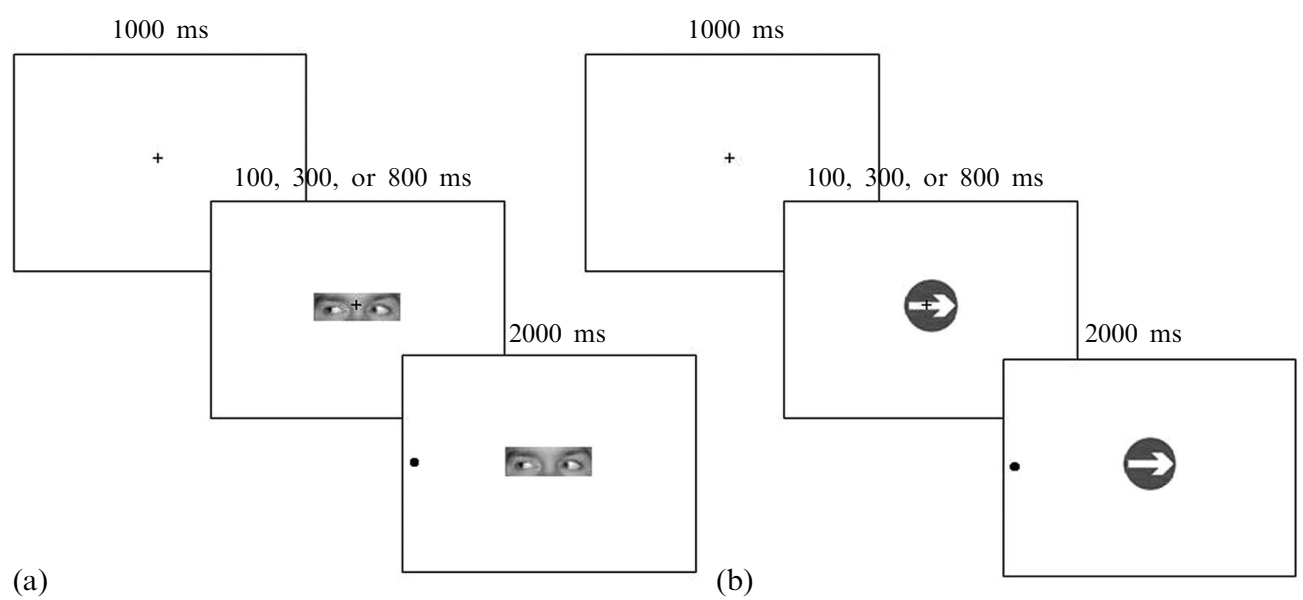

Figure 2. (a) Procedure for a congruent trial with eye gaze cues. A fixation cross is displayed for $1000 \mathrm{~ms}$ after which the cue is displayed. After an SOA of 100,300 , or $800 \mathrm{~ms}$ the 'target' is presented to the left or right of the cue and remains on-screen for $2000 \mathrm{~ms}$. The correct direction for the anti-saccade is in the opposite direction to the 'target'. There is an intertrial interval of $1000 \mathrm{~ms}$. (b) Procedure for an incongruent trial with arrow cues. The figure is not drawn to scale. 
2.1.4 Data analysis. Saccade parameters were extracted off-line with the Dataviewer software (SR Research, Canada). Saccades were detected as those eye movements which exceeded a velocity of $8000 \mathrm{deg} \mathrm{s}^{-1}$ and an acceleration of $30 \mathrm{deg} \mathrm{s}^{-1}$. The saccade given an index of 1 by Dataviewer was taken as the response for each trial for each participant. Of these saccades, only those which were executed towards the left or the right were retained for further analysis. Of these left and right responses, those with latencies between 80-699 ms (a range suggested to omit anticipations and delayed responses; see Fischer et al 1997), an amplitude of more than $2.0 \mathrm{deg}$, and which began in the central region of the screen were retained and the remainder of the trials were removed. This data preparation procedure (removal of trials based on the directional, latency, amplitude, and start position criteria) removed $1.53 \%$ of the data. Of these remaining saccades, those which were initiated in the direction opposite to the peripheral stimulus were considered as correct responses, while those made in the same direction of the peripheral stimulus were classed as errors.

\subsection{Results}

2.2.1 Saccadic reaction time. The mean correct SRT for each participant in each condition was calculated. SPSS version 15 was used to perform a three-way repeated-measures ANOVA on these data, with 2 levels on the factor cue type (arrows, eyes), 3 levels on the factor SOA $(100,300,800 \mathrm{~ms})$, and 3 levels on the factor congruency (congruent, neutral, incongruent). There was a main effect of SOA $\left(F_{2,26}=34.85, p<0.001\right.$; $\mathrm{H}-\mathrm{F}$ criterion), with response times decreased at $800 \mathrm{~ms}(M=245.64 \mathrm{~ms}, \mathrm{SD}=8.00 \mathrm{~ms})$ SOA relative to at $100 \mathrm{~ms}(M=282.64 \mathrm{~ms}, \mathrm{SD}=7.34 \mathrm{~ms})$ and $300 \mathrm{~ms}(M=264.89 \mathrm{~ms}$, $\mathrm{SD}=6.81 \mathrm{~ms})$ SOAs. There was also a main effect of congruency $\left(F_{1,13}=9.47\right.$, $p<0.001)$, with congruent trials producing the fastest responses $(M=258.96 \mathrm{~ms}$, $\mathrm{SD}=7.00 \mathrm{~ms})$, followed by incongruent trials $(M=264.30 \mathrm{~ms}, \mathrm{SD}=7.31 \mathrm{~ms})$, with the slowest responses on neutral trials $(M=269.91 \mathrm{~ms}, \mathrm{SD}=6.98 \mathrm{~ms})$. Planned comparisons showed that congruent trials were quicker than neutral $\left(F_{1,13}=20.24, p=0.001\right)$ and that incongruent trials were also quicker than neutral $\left(F_{1,13}=5.60, p=0.034\right)$, but the difference between congruent and incongruent did not reach significance $\left(F_{1,13}=3.85, p=0.072\right)$. There was no main effect of cue type, but this factor did interact with SOA $\left(F_{2,26}=4.81, p=0.017\right)$, indicating that the effects of the cues on reaction time changed over time. However, paired-samples $t$-tests conducted between gaze and arrow pairs at each SOA, collapsed over congruency, showed that only at $100 \mathrm{~ms}$ SOA did the difference in SRT between gaze and arrows approach significance $\left(100 \mathrm{~ms}: t_{13}=2.09, p=0.057 ; 300 \mathrm{~ms}: t_{13}=0.092, p=0.372 ; 800 \mathrm{~ms}\right.$ : $\left.t_{13}=0.12, p=0.904\right)$. In addition there was an SOA by congruency interaction $\left(F_{4,52}=2.59, p=0.049\right)$, indicating that there was some change in the congruency effects over the three SOAs, but as interaction was only marginally significant, further tests were not conducted.

Having been intended as a baseline with which to compute the relative costs and benefits associated with incongruent and congruent cues, the finding above that these trials produced the longest SRTs, was unexpected. Although interesting in itself and as such will be discussed later, the influence of the neutral cues may have masked differences between the effects of congruent and incongruent cues on SRTs, which was the comparison of greatest interest as it measures the relative directional effects of the cues on specifically spatial attention mechanisms. For this reason a second $(2 \times 3 \times 2)$ repeated-measures ANOVA was conducted which excluded the data from neutral trials in order to isolate and identify any differentiation between the directional effects of the gaze and arrow cues on saccadic orienting. 
The removal of the neutral trials had a marked effect on the results. The main effect of SOA remained $\left(F_{2,26}=27.75, p<0.001 ; \mathrm{H}-\mathrm{F}\right.$ criterion). However, the main effect of congruency did not reach significance $\left(F_{1,13}=3.85, p=0.072\right)$ indicating that the neutral trials were largely responsible for the main effect of congruency in the first analysis. The cue by SOA interaction was still significant $\left(F_{1,13}=3.58, p=0.042\right)$. Importantly, a new interaction emerged between cue and congruency $\left(F_{1,13}=8.16, p=0.014\right)$, indicating that there was a difference between the effects of the congruent and incongruent arrow and gaze cues on reaction times. Paired-samples $t$-tests were conducted on gaze and arrow congruent/incongruent pairs, collapsed over SOA. For gaze cues, SRTs on congruent trials were significantly quicker than on incongruent trials $\left(t_{13}=2.92\right.$, $p=0.012$ ), but for arrow cues there was no difference between congruent and incongruent trials $\left(t_{13}=0.59, p=0.568\right)$. Figure 3 shows the correct SRTs for congruent, neutral, and incongruent gaze and arrow cues over the three SOAs (also see table 1), whilst figure 4 shows the significant interaction between cue type and congruency, collapsed over SOA, in the analysis without the neutral data.

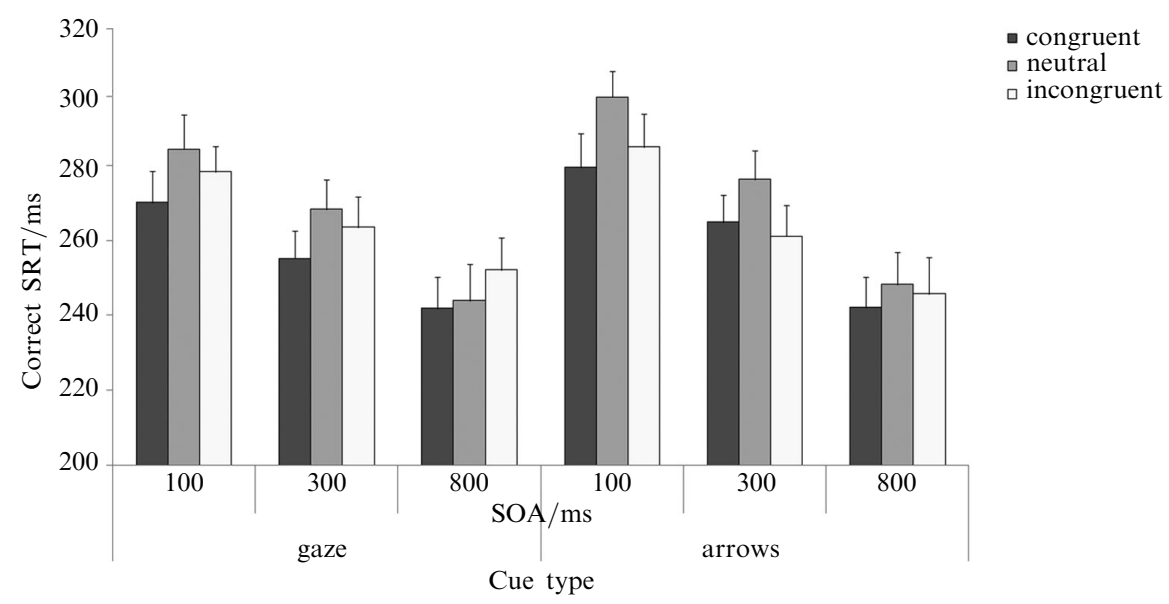

Figure 3. Mean correct SRTs for congruent, neutral, and incongruent trials over the three SOAs for gaze and arrow cues in experiment 1. Error bars represent standard error of the mean.

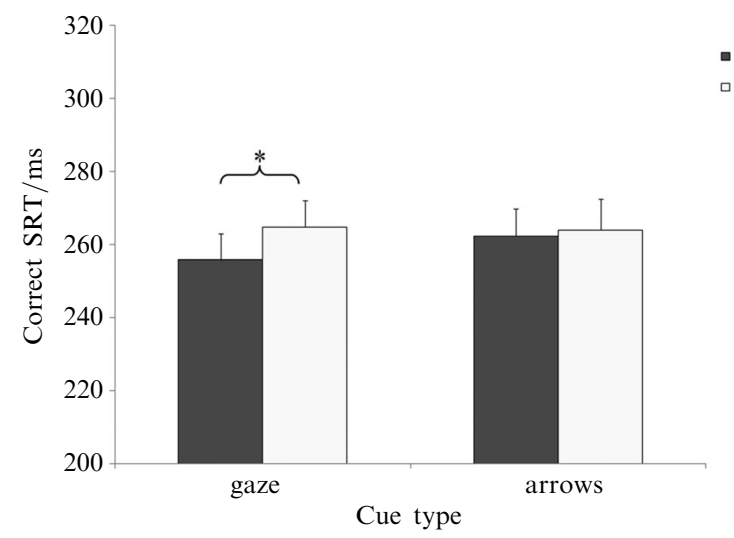

- congruent

$\square$ incongruent

2.2.2 Error rates. In 455 trials (10.68\% of the total completed trials), the first response was an error: a saccade incorrectly initiated towards the target. The percentage of errors committed in each condition was calculated for each participant by dividing the number of errors committed by the total number of trials for each condition, converted to a percentage. 
Table 1. Means and standard errors of all conditions in experiment 1 (gaze and arrows), experiment 2 (words), and experiment 3 (pointing). Note: A neutral condition was not included in experiment 3.

\begin{tabular}{|c|c|c|c|c|c|c|c|}
\hline \multirow[t]{2}{*}{ Conditions } & \multirow[t]{2}{*}{$\mathrm{SOA} / \mathrm{ms}$} & \multicolumn{2}{|c|}{ Congruent } & \multicolumn{2}{|c|}{ Neutral } & \multicolumn{2}{|c|}{ Incongruent } \\
\hline & & $M$ & $\mathrm{SE}$ & $M$ & SE & $M$ & SE \\
\hline \multirow[t]{3}{*}{ Gaze } & 100 & 270.12 & 8.42 & 284.44 & 9.31 & 278.39 & 6.81 \\
\hline & 300 & 255.17 & 7.44 & 268.33 & 7.98 & 263.50 & 8.13 \\
\hline & 800 & 241.82 & 8.32 & 243.86 & 9.83 & 252.13 & 8.58 \\
\hline \multirow[t]{3}{*}{ Arrows } & 100 & 279.58 & 9.05 & 298.35 & 7.03 & 284.97 & 8.91 \\
\hline & 300 & 264.92 & 7.34 & 276.29 & 7.85 & 261.12 & 8.27 \\
\hline & 800 & 242.12 & 8.23 & 248.20 & 8.71 & 245.70 & 9.75 \\
\hline \multirow[t]{3}{*}{ Words } & 100 & 265.13 & 8.75 & 272.18 & 6.80 & 270.49 & 9.73 \\
\hline & 300 & 252.60 & 7.02 & 252.95 & 7.69 & 253.18 & 6.07 \\
\hline & 800 & 235.24 & 6.41 & 240.69 & 5.98 & 239.88 & 6.57 \\
\hline \multirow[t]{3}{*}{ Pointing } & 100 & 275.51 & 8.90 & - & - & 306.44 & 7.53 \\
\hline & 300 & 261.30 & 5.60 & - & - & 272.52 & 8.38 \\
\hline & 800 & 242.55 & 5.55 & - & - & 247.90 & 6.00 \\
\hline
\end{tabular}

An initial three-way repeated-measures ANOVA was conducted on these data with 2 levels on the factor cue type (arrows, eyes), 3 levels on the factor SOA (100, 300, $800 \mathrm{~ms}$ ), and 3 levels on the factor congruency (congruent, neutral, incongruent). The main effect of SOA was significant $\left(F_{2,26}=15.53, p<0.001 ; \mathrm{H}-\mathrm{F}\right.$ criterion $)$, with most errors executed at the $100 \mathrm{~ms}$ SOA and the least at $800 \mathrm{~ms}$ SOA. There was an interaction between cue type and congruency $\left(F_{2,26}=4.91, p=0.016\right)$, with neutral gaze cues producing the most errors relative to incongruent and congruent, but with neutral arrow cues resulting in less errors than the other congruencies. As was apparent with the analyses of SRT, the neutral trials unexpectedly influenced the results which had the effect of obscuring the directional influences of the cues on congruent versus incongruent trials. To investigate the relative directional effects of congruent and incongruent cues on error rates, a second ANOVA was conducted without the neutral trial data. The interaction became non-significant $(F<1.0, p=0.747)$, indicating that it was the difference in the effects of the neutral gaze and arrow cues, rather than the difference between congruent and incongruent cues, which was responsible for the interaction in the initial analysis. No other main effects or interactions reached significance, in either the analysis with or without neutral trials.

\subsection{Discussion}

The results from experiment 1 showed that, in an anti-saccade task, gaze cues but not arrows facilitate saccadic response latencies in the opposite direction to the cues. This finding replicated that of Koval et al (2005) in terms of SRT, but effects on error rates were non-significant in the current experiment. Nevertheless, the results argue against the gaze imitation hypothesis, which suggests that imitative eye movements are automatically programmed in observers on perceiving another's averted eye gaze. It further demonstrates that gaze cues have a greater influence on the oculomotor system than arrows, given a sufficiently challenging task, with arrow cues appearing to be easy to ignore under increased cognitive demands. In addition, the interaction between cue type and SOA indicates that gaze cues may have an overall facilitatory effect on SRTs at short SOAs relative to arrow cues.

An unanticipated result in experiment 1 related to the neutral cues. It was expected that the SRTs and error rates on neutral trials would lie between those on congruent and incongruent trials. However, this was not so. Specifically, it appeared that both gaze and arrow neutral cues (images of eyes closed and the mini-roundabout road sign, respectively) had inhibitory effects on SRTs in both congruent and incongruent conditions. 
In terms of error rates, the neutral arrow cues also appeared to inhibit the production of erroneous saccades to a greater extent than either congruent or incongruent cues, but this effect was not present with gaze cues. It therefore appeared that the novel stimuli chosen to represent the neutral conditions in the current experiment had a general engaging effect on the saccadic system, which acted to inhibit the production of saccades relative to the directional versions of the cues. It is curious that this should be so, particularly for the eyes-closed neutral gaze cues, which had been chosen in favour of direct gaze cues which are known to actively engage attention, particularly to avoid this possibility (Senju and Hasegawa 2005). Although certainly an intriguing result, and one worthy of future investigation, the primary measure of interest had been the difference in response times on congruent and incongruent trials which provides a measure of the spatial influence of the directional cues on saccadic orienting. It was for this reason that a second analysis of variance, excluding data from the neutral condition, was conducted. In experiment 2, a neutral condition was again included, but this time a stimulus was chosen which was anticipated to differ from the left/right cues only in terms of its directionality.

\section{Experiment 2}

In Hodgson et al's (2009) oculomotor Stroop task, the directional meaning of words presented in different ink colours automatically recruited the saccadic system, despite participants being instructed to ignore the identity of the word. Furthermore, several studies of covert attentional orienting have shown that task-irrelevant word cues can shift attention in the cued direction (Ho and Spence 2006; Hommel et al 2001). Therefore experiment 2 expanded upon these studies by investigating the influence of directional word cues (LEFT and RIGHT) which were entirely task-irrelevant within the same anti-saccade task used in experiment 1. Based on the previous studies of covert and overt attentional orienting, one possible outcome of experiment 2 was that the cues would influence saccadic responses, even though they were to be ignored. Whether this predicted facilitation would be in the direction of the cues or in the opposite direction (as was seen with gaze cues in experiment 1) was not obvious. However, experiment 1 demonstrated that, although familiar directional stimuli, nonbiological arrow cues had very little influence on saccadic responses in the anti-saccade task. Therefore, an alternative outcome of experiment 2 was that the equally nonbiological word cues may have a comparably negligible influence on saccadic responses to arrows, with the cognitive complexity of the anti-saccade task overriding any automatic effects of the word cues which might otherwise become evident in less demanding tasks.

As in experiment 1, neutral cues were again used in experiment 2. As the stimuli used for the left, right, and neutral cues were all comprised of strings of letters in an identical, uniform colour and presented as images of the same size, it was anticipated that the neutral cues should now provide a baseline against which the effects of congruent and incongruent cues could be assessed.

\subsection{Methods}

3.1.1 Participants. A further fourteen healthy naive English speaking undergraduates from the School of Psychology, University of Exeter (twelve female) aged between 18 and 27 years $(M=20.00$ years; $\mathrm{SD}=2.77$ years $)$ took part in this experiment in exchange for course credit. All participants had either normal or corrected-to-normal vision. Written informed consent was given by all participants, and ethical approval for this experiment was granted by the Ethics Committee of the School of Psychology, University of Exeter. The research was carried out in accordance with The Code of Ethics of the World Medical Association (Declaration of Helsinki). 
3.1.2 Apparatus and stimuli. Apparatus was identical to that in experiment 1 . The directional word cues were prepared in Corel Paint Shop Pro X and were comprised of the letter strings LEFT, RIGHT, and XXXX (the neutral condition) written in Arial font, 130 points, in bold. To match the perceptual properties of the stimuli as closely as possible to those used in the previous experiments, the colour of the cues matched that of the road signs used in experiment 1 and had the RGB value of $0,122,194$, a mid-blue. The image canvas of the word cues was the same size as the eye-gaze cues used in experiment 1, resulting in cues which subtended $5.52 \mathrm{deg}$ of visual angle.

3.1.3 Procedure. The procedure was similar to that used in experiment 1, with the exception that, as there was only one type of cue, only three blocks of 60 trials were presented to each participant, resulting in each participant completing 180 trials.

3.1.4 Data preparation and analysis. Completed trials were isolated by using the same criteria as in experiment 1 . This procedure removed 176 trials representing $6.11 \%$ of trials.

\subsection{Results}

3.2.1 Saccadic reaction time. A two-way repeated-measures ANOVA was conducted on the SRT data with 3 levels on the factor SOA (100, 300, $800 \mathrm{~ms})$ and 3 levels on the factor congruency (congruent, neutral, incongruent). There was a significant main effect of $\operatorname{SOA}\left(F_{1.33,17.32}=32.56, p<0.001 ; \mathrm{H}-\mathrm{F}\right.$ criterion), with SRTs decreasing as SOAs increased. The main effect of congruency did not reach significance $\left(F_{2,26}=2.89\right.$, $p=0.074)$. The interaction was also not significant $(F<1.00)$. A second ANOVA was conducted which excluded the data from neutral trials as in experiment 1 . There was a similar main effect of SOA $\left(F_{1.44,18.74}=20.89, p<0.001 ; \mathrm{H}-\mathrm{F}\right.$ criterion $)$, but the effect of congruency was not significant $\left(F_{1,13}=2.81, p=0.12\right)$. The interaction did not approach significance $(F<1.00)$. Figure 5 shows the mean correct SRTs for all conditions in experiment 2 , which are also represented in table 1.

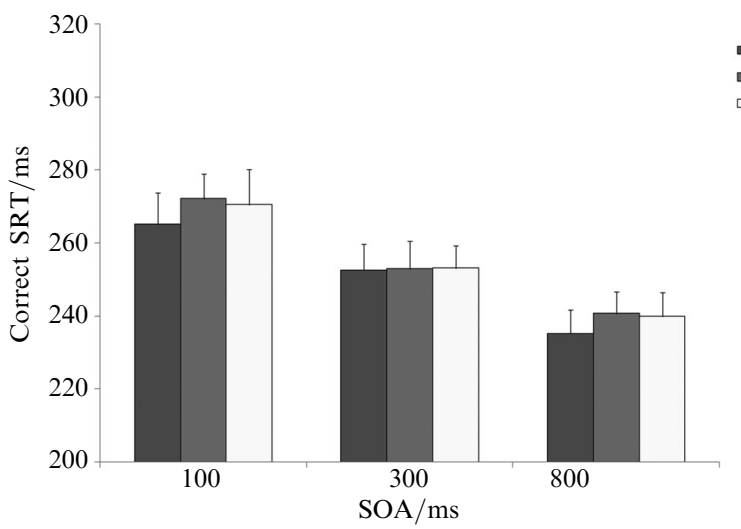

- congruent
neutral
$\square$ incongruent

Figure 5. Mean correct SRTs for congruent, neutral, and incongruent word cues over the three SOAs in experiment 2. Error bars represent standard error of the mean. None of the main effects or interactions were significant in experiment 2 .

3.2.2 Error rates. A total of 201 errors were committed, accounting for $8.58 \%$ of the completed trials.

A two-way ANOVA was conducted on the percentage of errors per condition per participant, with 3 levels on the factor SOA $(100,300,800 \mathrm{~ms})$ and 3 levels on the factor congruency (congruent, neutral, incongruent).

There was a significant main effect of SOA $\left(F_{1.27,16.52}=15.82, p<0.001 ; \mathrm{H}-\mathrm{F}\right.$ criterion), with the majority of errors being committed at $100 \mathrm{~ms}$ SOA $(M=16.82 \%$, $\mathrm{SE}=4.04 \%)$ with similar percentages executed at $300 \mathrm{~ms} \mathrm{SOA}(M=5.12 \%, \mathrm{SE}=1.93 \%)$ and $800 \mathrm{~ms}$ SOA $(M=3.38 \%, \mathrm{SE}=0.98 \%)$. The main effect of congruency did not reach significance nor did the interaction $(F \mathrm{~s}<1.00)$. 


\subsection{Discussion}

Experiment 2 demonstrated that in the anti-saccade task, task-irrelevant directional word cues have very little influence on saccadic orienting, in terms of SRT or error rates (with only a trend-level effect of congruency on SRT emerging when the neutral trials were included in the analysis). This finding contrasts with previous work using covert attentional cueing paradigms, which have shown that, to-be-ignored, word cues cause manual reaction times to be reduced for congruent targets (Ho and Spence 2006; Hommel et al 2001). It further contrasts with the findings of Hodgson et al (2009) who demonstrated that the direction of saccades was influenced by the, to be ignored, identity of words in an oculomotor Stroop task. It is possible that in the current experiment, the increased cognitive demands of the anti-saccade task counteracted the automatic processing of the word cues, and this is why congruency effects were not seen here.

The results of experiments 1 and 2 have shown that only task-irrelevant socially and biologically relevant gaze cues are capable of influencing saccadic orienting in the anti-saccade task, whereas non-biological arrow and word cues do not. In order to test whether the biological nature of stimuli is the reason that gaze cues have this effect on oculomotor responses, experiment 3 explored the influence of photographic finger-pointing cues on performance in the anti-saccade task.

\section{Experiment 3}

\subsection{Materials and methods}

4.1.1 Participants. A different group of seventeen healthy undergraduates and postgraduates from the University of Exeter (twelve female) aged between 18 and 38 years ( $M=21.35$ years, $\mathrm{SD}=3.61$ years) took part in this experiment in exchange for $£ 4$. All participants had either normal or corrected-to-normal vision. Written informed consent was given by all participants and ethical approval for this experiment was granted by the Ethics Committee of the School of Psychology, University of Exeter. The research was carried out in accordance with The Code of Ethics of the World Medical Association (Declaration of Helsinki).

4.1.2 Apparatus and stimuli. Apparatus was identical to that used in experiments 1 and 2. The finger-pointing cues were prepared in Corel Paint Shop Pro X and were comprised of photographs of a hand pointing left or right on a white background. The right hand cue was a mirror image of the photograph taken for the left hand cue to control for variations in stimulus properties. The image canvas of the pointing cues was the same size as the eye-gaze cues used in experiment 1 , resulting in cues which subtended $5.52 \mathrm{deg}$ of visual angle. As the neutral cues in experiment 1 appeared not to act as the baseline with which to compare costs and benefits of congruent and incongruent cues as they had been intended, and due to the difficulty in identifying an appropriate stimulus to act as a neutral finger-pointing cue, neutral cues were omitted from this experiment.

4.1.3 Procedure. The procedure was similar to that used in the previous experiments. As there were no neutral cues and only one cue type, participants completed 120 trials split into two blocks.

4.1.4 Data preparation and analysis. The data preparation procedure removed 127 trials representing $6.23 \%$ of the total number of trials.

\subsection{Results}

4.2.1 Saccadic reaction time. The mean correct SRT for each participant in each condition was calculated. A two-way repeated-measures ANOVA was performed on correct SRTs, 3 levels on the factor SOA $(100,300,800 \mathrm{~ms})$, and 2 levels on the factor congruency (congruent, incongruent). 
There was a significant main effect of SOA $\left(F_{2,32}=44.04, p<0.001 ; \mathrm{H}-\mathrm{F}\right.$ criterion $)$, with responses getting faster as SOA increased. There was also a main effect of congruency $\left(F_{1,16}=56.75, p<0.001\right)$, with congruent trials significantly faster than incongruent (congruent: $M=259.79 \mathrm{~ms}, \mathrm{SE}=5.86 \mathrm{~ms}$; incongruent: $M=275.62 \mathrm{~ms}$, $\mathrm{SE}=6.72 \mathrm{~ms}$ ). The interaction between SOA and congruency was also significant $\left(F_{2,32}=9.92, p<0.001\right)$. Paired-samples $t$-tests demonstrated that this was due to SRTs on congruent trials being significantly faster than incongruent trials at the 100 and $300 \mathrm{~ms}$ SOAs, with a particularly large and highly significant difference at $100 \mathrm{~ms}$ SOA (100 ms: $\left.t_{16}=7.18, p<0.001 ; 300 \mathrm{~ms}: t_{16}=2.53, p=0.022\right)$, but that there was no difference between SRTs on congruent and incongruent trials at $800 \mathrm{~ms}$ SOA $\left(t_{16}=1.60, p=0.129\right.$; figure 6$)$. The correct SRTs for all conditions in experiment 3 can be seen in table 1 .

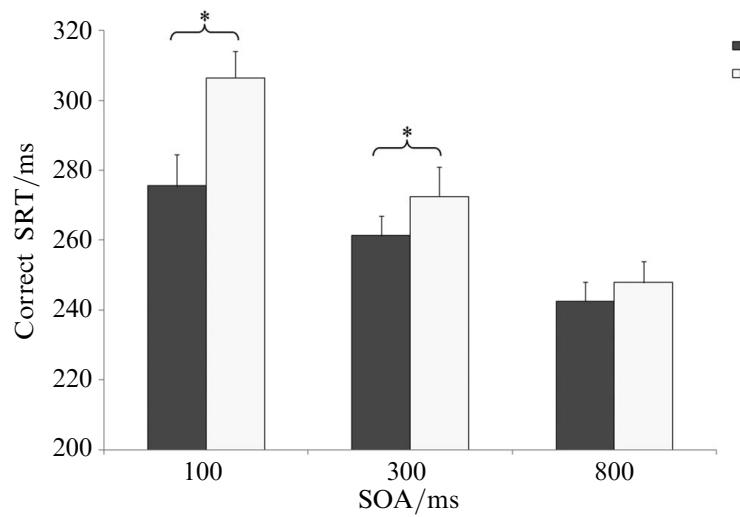

- congruent
$\square$ incongruent

4.2.2 Error rates. A total of 96 errors were committed, accounting for $4.70 \%$ of the completed trials.

A two-way ANOVA was conducted on the percentage of errors per condition per participant, with 3 levels on the factor SOA $(100,300,800 \mathrm{~ms})$ and 2 levels on the factor congruency (congruent, incongruent).

There was a main effect of $\operatorname{SOA}\left(F_{1.45,23.16}=5.41, p=0.019 ; \mathrm{H}-\mathrm{F}\right.$ criterion), with error rates decreasing as SOA increased, reflecting the enhanced ability to voluntarily suppress saccades as preparation time increased. There was no significant effect of congruency, however $\left(F_{1,16}=2.14, p=0.163\right)$. The interaction was not significant.

\section{Cross-experiment analysis}

In order to identify whether biological cues (gaze and pointing) as a category are processed preferentially by the oculomotor system, it was suggested by one anonymous reviewer that a cross-experiment analysis would be necessary. Although somewhat complicated by the slight difference in experimental design across the three experiments, such an analysis was conducted.

A mixed-design ANOVA was carried out, with 2 levels on the between-subjects factor cue category (biological, non-biological), 3 levels on the within-subjects factor SOA (100, $300,800 \mathrm{~ms}$ ), and 2 levels on the within-subjects factor congruency (congruent, incongruent). There was a significant main effect of SOA $\left(F_{1.91,108.61}=104.46, p<0.001, \mathrm{H}-\mathrm{F}\right.$ criterion) and a significant main effect of congruency $\left(F_{1,57}=34.81, p<0.001\right)$, but the main effect of cue category was not significant $(F<1.00)$. The interaction between cue category and congruency was highly significant $\left(F_{1,57}=15.08, p<0.001\right)$, with an overall mean congruency advantage for biological cues of $12.73 \mathrm{~ms}$, but only $2.63 \mathrm{~ms}$ with non-biological cues. The SOA by congruency interaction was also significant $\left(F_{1,57}=4.76, p=0.010\right.$, with a $13.03 \mathrm{~ms}$ congruency effect at $100 \mathrm{~ms}$ SOA but effects 
of only $4.15 \mathrm{~ms}$ at $300 \mathrm{~ms} \mathrm{SOA}$ and $5.85 \mathrm{~ms}$ at $800 \mathrm{~ms}$ ). However, the three-way interaction was not significant $\left(F_{2,114}=1.96, p=0.146\right)$.

SRT data for all conditions of the three experiments are presented in table 1.

\section{General discussion}

The current work set out to examine the influence of biological and non-biological directional cues on saccadic orienting. The findings extend previous research by comparing the effect of biological (eye gaze and finger pointing) and non-biological (arrows and words) cues on anti-saccade task performance. Although previous studies using simple saccadic tasks found that both gaze and arrow cues facilitate saccadic orienting (Kuhn and Benson 2007; Kuhn and Kingstone 2009; Kuhn et al 2010), the present findings, using a more complex task, suggest that socially relevant biological cues have privileged access to the oculomotor system.

In experiment 1 , eye gaze but not arrow cues caused participants to make faster anti-saccades in the opposite direction to the cues. This occurred despite the cues being non-predictive of target location and occurred even at short SOAs. The word cues in experiment 2 had no influence on saccades, whereas finger pointing cues (experiment 3 ) also enhanced responding in the opposite direction to the cues. These findings extend those of Koval et al (2005), who examined the influence of gaze cues in the anti-saccade task and also found that participants responded more quickly on congruent trials. As Koval et al did not compare the effect of gaze cues with any other directional stimuli it was not possible to contextualise their findings in terms of 'social attention' per se. However, the current work demonstrates that biological cues influence saccadic responses in the anti-saccade task, whereas cues with no biological significance do not.

The demonstration of an automatic influence of biological finger pointing cues on saccades dovetails with recent work showing that to be ignored finger pointing cues also facilitate covert attention in the cued direction (Ariga and Watanabe 2009; Sato et al 2009, 2010; Tomonaga and Imura 2009). Pointing cues in the current study caused a larger congruency advantage than gaze cues only at the shorter SOAs, whereas the gaze cues influenced responses across the range of SOAs. The rapid emergence of a congruency effect for both gaze and pointing cues suggests that both act automatically on the saccadic system. For gaze cues, however, the fact that this effect persists even at the longest SOA suggests an inability to override their influence via top-down control. In this sense, gaze cues appear harder to ignore than pointing cues.

There are several possible explanations for the larger congruency effect observed with pointing cues. First, it may result from the configuration of the stimuli themselves. Pointing cues are more asymmetric than gaze cues and so their visual properties may explain why they exert a greater directional influence on response times. However, this seems unlikely as the arrow cues used in experiment 1 were more asymmetric than gaze cues, but exerted a smaller effect. A further possibility is that gaze and pointing cues may exert their attentional orienting effects via separate neural mechanisms, as has been suggested by Bayliss et al (2011). Using a gaze adaptation paradigm (see Jenkins et al 2006), these authors showed that adaptation via repeated exposure to leftward and rightward pointing gaze stimuli influenced subsequent gaze cueing in a covert target-detection task, whilst adaptation to pointing cues did not. On the basis of these findings they argued for the presence of a direct pathway for attentional orienting in response to gaze cues which is separate from that used by finger and arrows cues. However, as this argument places arrows and finger pointing in the same category, it is unable to account for the differential effects of arrows and finger pointing cues in the present study. Another explanation is that methodological differences between experiments 1 and 3 resulted in the different magnitudes of cueing effect. In experiment 1 , both gaze and arrow cues were mixed with neutral versions of the cues, whilst in 
experiment 3 neutral cues were not employed. Because of this, direct comparisons between the exact magnitude of the congruency effects across experiments ought to be made with some caution.

The finding that directional word cues did not influence performance in the antisaccade task is at odds with findings from covert attention studies (Ho and Spence 2006; Hommel et al 2001) and Hodgson et al's (2009) oculomotor Stroop task. However, unlike in Hodgson et al's task, where the word cues were task-relevant (the identity of the word was to be ignored but the font colour of the word was to be attended to), in the current work the word cues were entirely task-irrelevant and participants were explicitly instructed to ignore them. Furthermore, Ho and Spence, and Hommel et al do not explicitly state that participants were instructed to ignore the cues. This suggests that, unlike gaze and pointing cues, word cues do not exert an obligatory effect on saccadic responses, but their influence is dependent upon the extent to which they are attended to and actively processed by participants.

The neutral gaze and arrow cues used in experiment 1 unexpectedly caused SRTs to be slower than those made in the presence of the directional versions of the cues. One explanation for this is that as well as exerting directional effects, the left and right cues also disengage the saccadic system more generally in preparation for an eye movement, regardless of whether they are congruent or incongruent. In this sense, neutral cues would not only lack directionality, but would also cause additional engagement of the saccadic system accounting for the slower SRTs observed. However, the finding that the neutral gaze and arrow cues had opposite effects on error rates suggests that the engagement hypothesis may not entirely explain the data presented here. Rather, they may highlight an inherent problem in the use of neutral cues as a baseline, when such stimuli are often chosen somewhat arbitrarily on the basis of subjective judgments of neutrality (Jonides and Mack 1984).

There was no evidence in the present study for directional biological cues influencing the direction of error saccades towards the peripheral stimulus in the anti-saccade task. The reason for this may relate to the statistical power of the analysis as participants made fewer errors than might be expected in a standard anti-saccade task (see Hutton 2008). As error saccades in the anti-saccade task are generally considered to be reflexive in nature (Everling et al 1998), it might be suggested that the gaze and finger-pointing cues were not influencing reflexive saccade generation, but that they did affect voluntary saccade generation (as reflected in correct anti-saccade latency). This finding contrasts with those of Kuhn and colleagues (Kuhn and Benson 2007; Kuhn and Kingstone 2009; Kuhn et al 2010) who found increased errors in the cued direction in their voluntary saccade task. One possibility is that the anti-saccade task requires enhanced attentional engagement in order to prevent errors towards peripheral stimuli (Everling and Munoz 2000; Everling et al 1999; Munoz and Wurtz 1992) which also tends to suppress execution of saccades in response to cues.

As it is well established that certain regions of the brain are preferentially activated by biological stimuli (Allison et al 2000; Hooker et al 2003; Pelphrey et al 2005; Tipper et al 2008), it may not seem surprising that such stimuli are more difficult to ignore and cause a more obligatory effect on saccades when compared to non-biological cues. However, the findings of the present research are at odds with other recent reports which find indistinguishable effects for biological and non-biological cues (Kuhn and Benson 2007; Kuhn and Kingstone 2009; Kuhn et al 2010). The reason for this discrepancy may be that under low cognitive demands, biological and non-biological cues act in a similar manner and it is only in a more complex task that differences such as those seen here are uncovered. Another explanation for the separable effects of the gaze and arrow cues in the current article is the ecological validity rather than the social-biological relevance of the cues. In the current experiments, photographic 
biological cues were used, whilst in most previous work, schematicised stimuli were employed. In short, it may not be surprising that arrow and gaze cues have the identical effects on saccadic orienting in such studies when their natural form has been adjusted to such an extent that they looked almost identical.

It should also be noted that arrow cues may have more biological relevance than is commonly assumed. For example, traditional road signs in the UK are known as fingerposts and were originally shaped as pointing hands. Over the centuries the hand was abstracted into an arrow such that signs taking the original finger form are now very scarce. This evolution suggests that arrows may in fact be highly schematicised pointing hands. Interestingly, the current work shows that arrows may not influence the oculomotor system as effectively as pointing fingers, suggesting that transport signage designers might consider reinstating traditional fingerposts to more effectively direct action and attention on the highway.

The basic finding that anti-saccades are facilitated in the direction opposite to a gaze or pointing cue appears superficially counterintuitive. However, not only has this phenomenon been reported elsewhere with gaze cues as already discussed (Koval et al 2005) but similar effects have also been observed with sudden onset peripheral ('exogenous') cues in the anti-saccade task. Weber et al (1998) presented 'pro-cues' in the anti-saccade task, which were sudden-onset peripheral cues presented in the correct location for the correct anti-saccade response. Importantly, these cues were $100 \%$ valid, with the validity of the cues predicted to enhance performance by giving participants prior knowledge of the correct direction in which to pre-programme their responses. However, Weber et al discovered that participants were slower to respond when the cues were presented, than when the experiment contained no cues at all. In a further experiment ('non-informative random-direction cues'), these authors presented cues which could appear on the same or the opposite side to the target. They found that cues presented on the same side as the anti-saccade target resulted in faster SRTs than when the cue was presented on the opposite side to the target. Therefore there appeared to be a consistent benefit for cues appearing on the same side as the target (see also Reuter et al 2006).

What mechanism might underlie this counterintuitive effect? One possibility is that the cue causes an automatic shift of covert attention to the cued direction, facilitating target detection on congruent trials. However, this explanation would be counter to the pre-motor theory of attention which argues strongly that preparing to make a saccade and orienting covert attention cannot be decoupled (Rizzolatti et al 1987, 1994; Sheliga et al 1994, 1997; but see Hodgson and Muller 1995), predicting a slowing of responses on congruent trials. An alternative explanation invokes the concept of an anti-saccade 'task set' (Hodgson et al 2004). In the anti-saccade task, participants may be adopting a general response set in which they initiate an 'opposite' response which is automatically transposed onto the cue stimuli.

\section{Conclusion}

In the current article, we have demonstrated that task-irrelevant, eye gaze, and finger pointing cues cause facilitation of correct SRTs in the opposite direction to the cues in the anti-saccade task, but that this does not occur with arrows or directional word cues. These findings strongly suggest that biological cues have privileged access to the oculomotor system over non-biological cues and that the anti-saccade task is a sufficiently complex oculomotor paradigm with which to study the influence of such stimuli on saccadic behaviour. 


\section{References}

Allison T, Puce A, McCarthy G, 2000 "Social perception from visual cues: role of the STS region" Trends in Cognitive Sciences $4267-278$

Ariga A, Watanabe K, 2009 "What is special about the index finger?": The index finger advantage in manipulating reflexive attentional shift" Japanese Psychological Research 51 258-265

Bayliss A P, Bartlett J, Naughtin C K, Kritikos A, 2011 "A direct link between gaze perception and social attention" Journal of Experimental Psychology: Human Perception and Performance $37634-644$

Bonato M, Priftis K, Marenzi R, Zorzi M, 2009 "Normal and impaired reflexive orienting of attention after central nonpredictive cues" Journal of Cognitive Neuroscience 21745 - 759

Driver J, Davis G, Ricciardelli P, Kidd P, Maxwell E, Baron-Cohen S, 1999 "Gaze perception triggers reflexive visuospatial orienting" Visual Cognition 6 509-540

Everling S, Dorris M C, Klein R M, Munoz D P, 1999 "Role of primate superior colliculus in preparation and execution of anti-saccades and pro-saccades" Journal of Neuroscience 19 $2740-2754$

Everling S, Dorris M C, Munoz D P, 1998 "Reflex suppression in the anti-saccade task is dependent on prestimulus neural processes" Journal of Neurophysiology $801584-1589$

Everling S, Fischer B, 1998 "The antisaccade: a review of basic research and clinical studies" Neuropsychologia $36885-899$

Everling S, Munoz D P, 2000 "Neuronal correlates for preparatory set associated with pro-saccades and anti-saccades in the primate frontal eye field" Journal of Neuroscience $20387-400$

Farroni T, Csibra G, Simion G, Johnson M H, 2002 "Eye contact detection in humans from birth" Proceedings of the National Academy of Sciences of the USA $999602-9605$

Farroni T, Massaccesi S, Pividori D, Johnson M H, 2004 "Gaze following in newborns" Infancy $539-60$

Fischer B, Gezeck S, Hartnegg K, 1997 "the analysis of saccadic eye movements from gap and overlap paradigms" Brain Research Protocols 2 47-52

Friesen C K, Kingstone A, 1998 "The eyes have it! Reflexive orienting is triggered by nonpredictive gaze" Psychonomic Bulletin \& Review $5490-495$

Friesen C K, Ristic J, Kingstone A, 2004 "Attentional effects of counterpredictive gaze and arrow cues" Journal of Experimental Psychology: Human Perception and Performance 30 319-329

Gibson B, Bryant T, 2005 "Variation in cue duration reveals top-down modulation of involuntary orienting to uninformative symbolic cues" Perception \& Psychophysics $67749-758$

Guitton D, Buchtel H A, Douglas R M, 1985 "Frontal lobe lesions in man cause difficulties in suppressing reflexive glances and in generating goal-directed saccades" Experimental Brain Research $58455-472$

Hallett P, 1978 "Primary and secondary saccades to goals defined by instructions" Vision Research $181279-1296$

Hallett P, Adams B D, 1980 "The predictability of saccadic latency in a novel voluntary oculomotor task" Vision Research $20329-339$

Ho C, Spence C, 2006 "Verbal interface design: Do verbal directional cues automatically orient visual spatial attention?" Computers in Human Behavior $22733-748$

Hodgson T L, Golding C, Molyva D, Rosenthal C R, Kennard C, 2004 "Eye movements during task switching: Reflexive, symbolic, and affective contributions to response selection" Journal of Cognitive Neuroscience 16318 - 330

Hodgson T L, Muller H J, 1995 "Evidence relating to premotor theories of visuospatial attention", in Eye Movement Research: Mechanisms, Processes and Applications Eds J M Findlay, $\mathrm{R}$ Walker, R Kentridge (Amsterdam: Elsevier)

Hodgson T L, Parris B A, Gregory N J, Jarvis T, 2009 "The saccadic Stroop effect: Evidence for involuntary programming of eye movements by linguistic cues" Vision Research 49 569-574

Hommel B, Pratt J, Colzato L, Godijn R, 2001 "Symbolic control of visual attention" Psychological Science $12360-365$

Hooker C, Paller K, Gitelman D, Parrish T, Mesulam M, Reber P, 2003 "Brain networks for analyzing eye gaze" Cognitive Brain Research $17406-418$

Hutton S B, 2008 "Cognitive control of saccadic eye movements" Brain and Cognition 68 327-340

Jenkins R, Beaver J D, Calder A J, 2006 "I thought you were looking at me: direction-specific aftereffects in gaze perception" Psychological Science 17 506-513

Jonides J, Mack R, 1984 "On the cost and benefit of cost and benefit" Psychological Bulletin 96 $29-44$

Koval M, Thomas B, Everling S, 2005 "Task-dependent effects of social attention on saccadic reaction times" Experimental Brain Research $167475-480$ 
Kuhn G, Benson V, 2007 "The influence of eye-gaze and arrow pointing distractor cues on voluntary eye movements" Perception \& Psychophysics $69966-971$

Kuhn G, Benson V, Fletcher-Watson S, Kovshoff H, McCormick C, Kirkby J, Leekam S, 2010 "Eye movements affirm: automatic overt gaze and arrow cueing for typical adults and adults with autism spectrum disorder" Experimental Brain Research $201155-165$

Kuyn G, Kingstone A, 2009 "Look away! Eyes and arrows engage oculomotor responses automatically" Attention Perception \& Psychophysics $71314-327$

Munoz D P, Everling S, 2004 "Look away: The anti-saccade task and the voluntary control of eye movement" Nature Reviews Neuroscience 5 218-228

Munoz D P, Wurtz R H, 1992 "Role of the rostral superior colliculus in active visual fixation and execution of express saccades" Journal of Neurophysiology $671000-1002$

Pelphrey K, Morris J, Michelich C, Allison T, McCarthy G, 2005 "Functional anatomy of biological motion perception in posterior temporal cortex: An fMRI study of eye, mouth and hand movements" Cerebral Cortex 15 1866-1876

Quadflieg S, Mason M F, Macrae C N, 2004 "The owl and the pussycat: Gaze cues and visuospatial orienting" Psychonomic Bulletin \& Review 11 826-831

Reuter B, Herzog E, Kathmann N, 2006 "Antisaccade performance of schizophrenia patients: Evidence of reduced task-set activation and impaired error detection" Journal of Psychiatric Research $40122-130$

Ricciardelli P, Bricolo E, Aglioti S M, Chelazzi L, 2002 "My eyes want to look where your eyes are looking: Exploring the tendency to imitate another individual's gaze" NeuroReport $132259-2264$

Ristic J, Friesen C K, Kingstone A, 2002 "Are eyes special? It depends on how you look at it" Psychonomic Bulletin \& Review $9507-513$

Ristic J, Kingstone A, 2006 "Attention to arrows: Pointing to a new direction" Quarterly Journal of Experimental Psychology 591921 - 1930

Rizzolatti G, Riggio L, Dascola I, Umilta C, 1987 "Reorienting attention across the horizontal and vertical meridians-evidence in favour of a premotor theory of attention" Neuropsychologia $2531-40$

Rizzolatti G, Riggio L, Sheliga B M, 1994 "Space and selective attention" Attention and Performance $15231-265$

Sato W, Kochiyama T, Uono S, Yoshikawa S, 2009 "Commonalities in the neural mechanisms underlying automatic attentional shifts by gaze, gestures, and symbols" NeuroImage 45 984-992

Sato W, Kochiyama T, Uono S, Yoshikawa S, 2010 "Automatic attentional shifts by gaze, gestures, and symbols" Psychologia $\mathbf{5 3} 27-35$

Senju A, Hasegawa T, 2005 "Direct gaze captures visuospatial attention" Visual Cognition 12 127-144

Sheliga B M, Craighero L, Riggio L, Rizzolatti G, 1997 "Effects of spatial attention on directional manual and ocular responses" Experimental Brain Research $114339-351$

Sheliga B M, Riggio L, Rizzolatti G, 1994 "Orienting of attention and eye-movements" Experimental Brain Research $98507-522$

Tipper C, Handy T, Giesbrecht B, Kingstone A, 2008 "Brain responses to biological relevance" Journal of Cognitive Neuroscience $\mathbf{2 0} 879-891$

Tipples J, 2002 "Eye gaze is not unique: Automatic orienting in response to uninformative arrows" Psychonomic Bulletin \& Review $9314-318$

Tipples J, 2008 "Orienting to counterpredictive gaze and arrow cues" Perception \& Psychophysics $7077-87$

Tomonaga M, Imura T, 2009 "Human gestures trigger different attentional shifts in chimpanzees (Pan troglodytes) and humans (Homo sapiens)" Animal Cognition 12 S11-S18

Weber H, Durr N, Fischer B, 1998 "Effects of pre-cues on voluntary and reflexive saccade generation. II. Pro-cues for anti-saccades" Experimental Brain Research $120417-431$ 


\section{PERCEPTION}

VOLUME 412012

www.perceptionweb.com

Conditions of use. This article may be downloaded from the Perception website for personal research by members of subscribing organisations. Authors are entitled to distribute their own article (in printed form or by e-mail) to up to 50 people. This PDF may not be placed on any website (or other online distribution system) without permission of the publisher. 